\title{
Portable Magnetofluidic Device for Point-of-Need Detection of African Swine Fever
}

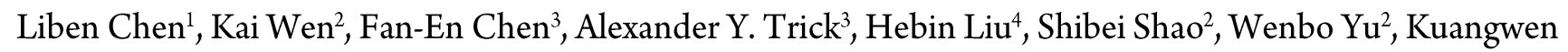

Hsieh $^{1}$, Zhanhui Wang ${ }^{2}$, Jianzhong Shen ${ }^{2}$, Tza-Huei Wang ${ }^{* 1,3,5}$

${ }^{1}$ Department of Mechanical Engineering, Johns Hopkins University, Baltimore, Maryland 21218, United States

${ }^{2}$ College of Veterinary Medicine, China Agricultural University, Beijing 100193, China

${ }^{3}$ Department of Biomedical Engineering, Johns Hopkins University, Baltimore, Maryland 21218, United States

${ }^{4}$ Beijing Mingrida Science \& Technology Development Co., Ltd, Beijing 100095, China

${ }^{5}$ Institute for NanoBiotechnology, Johns Hopkins University, Baltimore, MD 21218, United States

\section{Corresponding Author}

* Tza-Huei Wang, Johns Hopkins University, 3400 North Charles Street, Croft Hall 200A, Baltimore, Maryland 21218, USA; email: thwang@jhu.edu

\section{Author Contributions}

L.C. and K.W. contributed equally to this work. The manuscript was written through the contributions of all authors. All authors have given approval to the final version of the manuscript.

\section{TABLE OF CONTENT}

Table s1. Specificity assessment.

Figure s1. Instrument design and graphical user interface

Figure s2. Cartridge design

Figure s3. On-cartridge DNA purification

Figure s4. Testing setup at point-of-need settings.

Figure s5. DNA recovery comparison between the magnetofluidic device and a commercial DNA extraction kit.

Figure s6. Ct determination using logistic fitting.

Figure s7. Sensitivity comparison of saliva and serum 
Table s1. Specificity assessment.

\begin{tabular}{lllc}
\hline Organism & Material type & Concentration & Results \\
\hline $\begin{array}{l}\text { Classical swine fever virus } \\
\begin{array}{l}\text { Porcine reproductive and respiratory } \\
\text { syndrome virus }\end{array}\end{array}$ & attenuated vaccine & $\geq 1 \times 10^{5} \mathrm{TCID} 50 / \mathrm{mL}$ & No amplification \\
$\begin{array}{l}\text { Porcine parvovirus } 2 \\
\text { Pseudorabies virus }\end{array}$ & cell culture & $\geq 1 \times 10^{5} \mathrm{TCID} 50 / \mathrm{mL}$ & No amplification \\
\hline
\end{tabular}
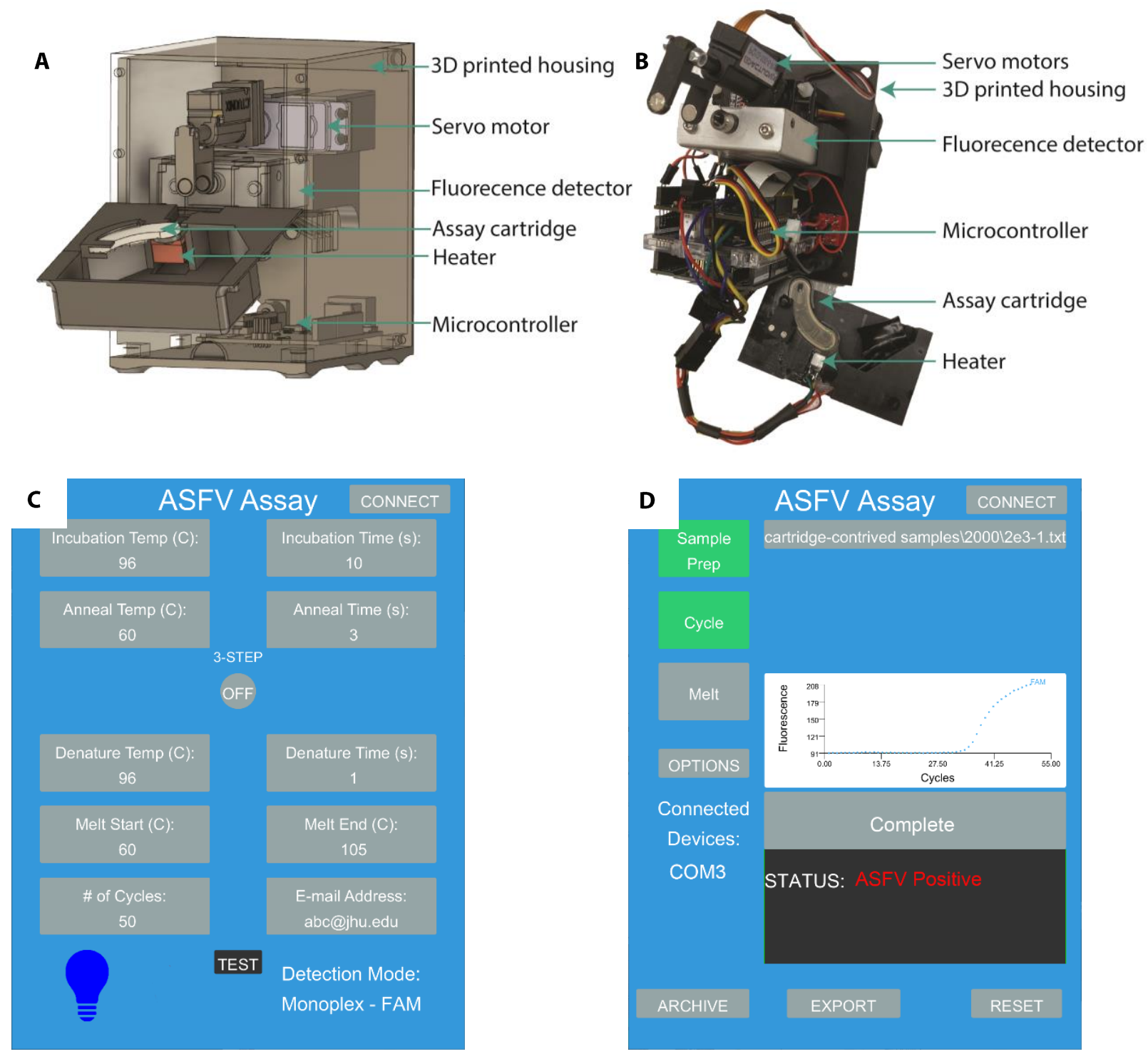

Figure s1. Instrument design and graphical user interface. (A) The 3-D computer-aided design model of the instrument illustrates that the instrument houses (i) a rotational servo motor and a linear servo motor for magnetic particle transfer, (ii) a fluorescence detector for real-time fluorescence detection, (iii) a miniaturized heating element for ultra-fast thermal cycling, (iv) a microcontroller for controlling magnetic manipulation, heating and cooling, fluorescence signal acquisition, and wireless communication with user terminals (such as tablets or smartphones). (B) A lab prototype of the device shows the simplicity of the instrument design. The 3-D printed housing was removed to reveal all the components within the device. (C) A 
screenshot of the graphical user interface shows the assay settings, which cover common functionalities that commercial PCR machines typically offer. (D) A screenshot of the graphical user interface shows the real-time monitoring and reporting of testing results.

A

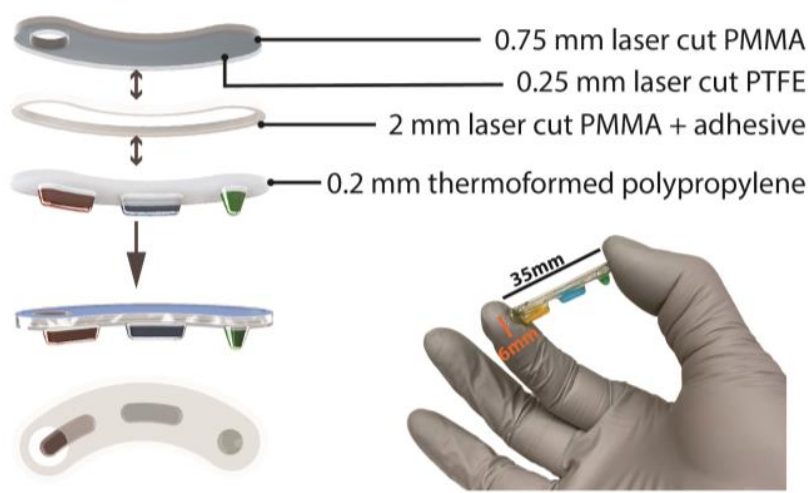

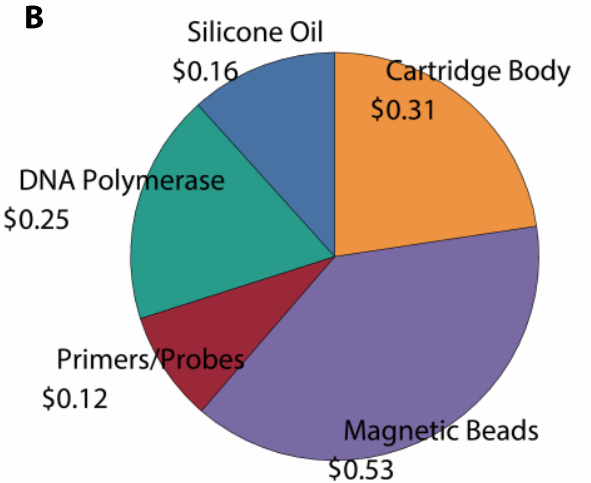

Figure s2. Cartridge design. (A) The assay cartridge features a laser-cut top layer of poly(methyl methacrylate) (PMMA) coated with poly(tetrafluoroethylene) tape for a smooth transfer of magnetic particles between wells, a middle laser-cut layer of PMMA as a spacer for containing silicone oil in the transition region between wells, and a bottom thermoformed polypropylene layer with extruded wells for containing reagents. The assay cartridge's simple thermoplastic design makes it highly cost-effective and amenable to fabrication by scalable manufacturing techniques (e.g. roll-to-roll lamination, thermoforming, die-cutting). (B) The cost of goods analysis shows a disposable cartridge costs $\sim \$ 1.37$, including reagents.



On-Cartridge DNA purification

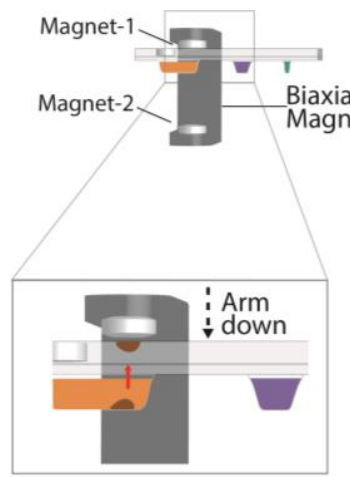

(i) Attract magnetic particles out to cartridge ceiling by magnet -1

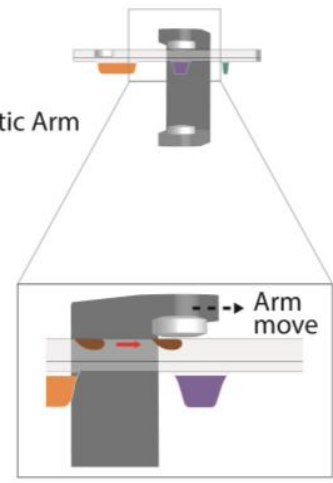

(ii) Transfer particles along cartridge ceiling by magnet-1

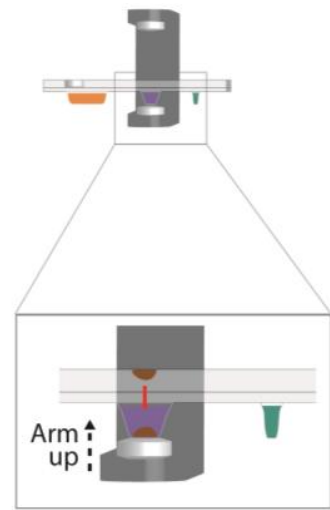

(iii) Pull down particles by magnet-2

Figure s3. On-cartridge DNA purification. The automated DNA extraction and purification are realized using ChargeSwitch ${ }^{\circledR}$ magnetic particles. Capture and release of nucleic acids from these particles are mediated by the $\mathrm{pH}$ of the respective reagents. In low $\mathrm{pH}$ solutions, the particles are positively charged for the capture of negatively charged nucleic acids; whereas, at higher $\mathrm{pH}$, the particle surface is neutralized to allow the release of any electrostatically bound analytes. Particle transfer (i, ii, iii) is actuated by a biaxial magnetic arm. 

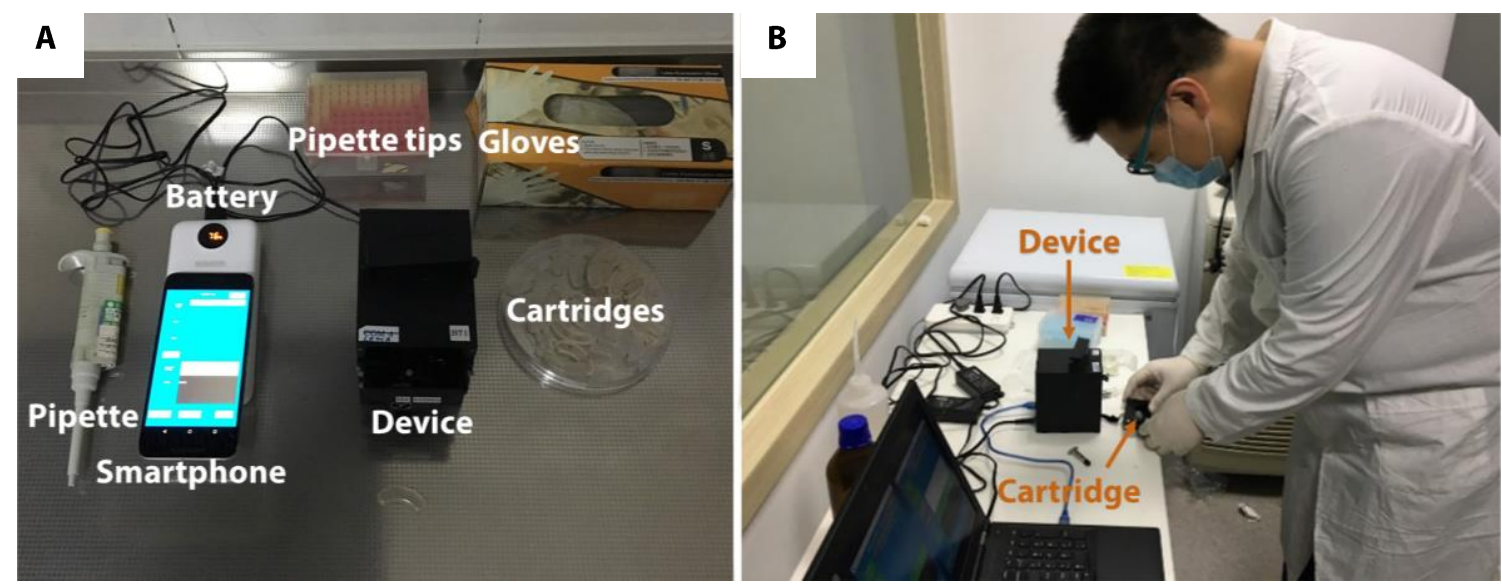

Figure s4. Testing setup at point-of-need settings. (A) Supplies needed for the magnetofluidic ASF testing include a pipette with compatible tips, a battery (if no power supply at the testing site), a smartphone, the magnetofluidic device, the cartridges, and personal protective equipment such as gloves. (B) An untrained lab technician was performing the magnetofluidic test.

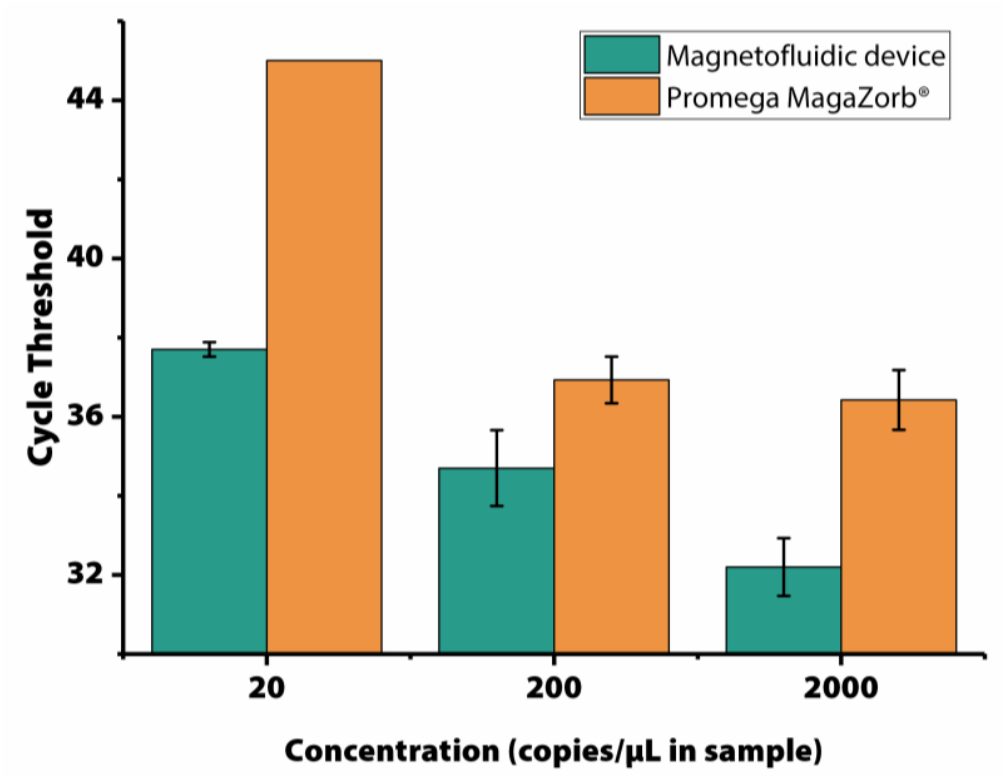

Figure s5. DNA recovery comparison between the magnetofluidic device and a commercial DNA extraction kit Promega MagaZorb DNA miniPrep. A series of known concentrations of synthetic ASFV DNA targets were spiked in $10 \mu \mathrm{L}$ of porcine serum. DNA extraction with the MagaZorb kit was performed according to the manufacturer's instructions. To maximize the recovery, a recommended minimum elution volume of $50 \mu \mathrm{L}$ was used to elute the DNA from the resin. A maximum of $4 \mu \mathrm{L}$ of DNA eluate was added to $10 \mu \mathrm{L}$ (to match with our magnetofluidic device) of PCR master mix for the final PCR detection on a BioRad CFX 96 real-time PCR System. 

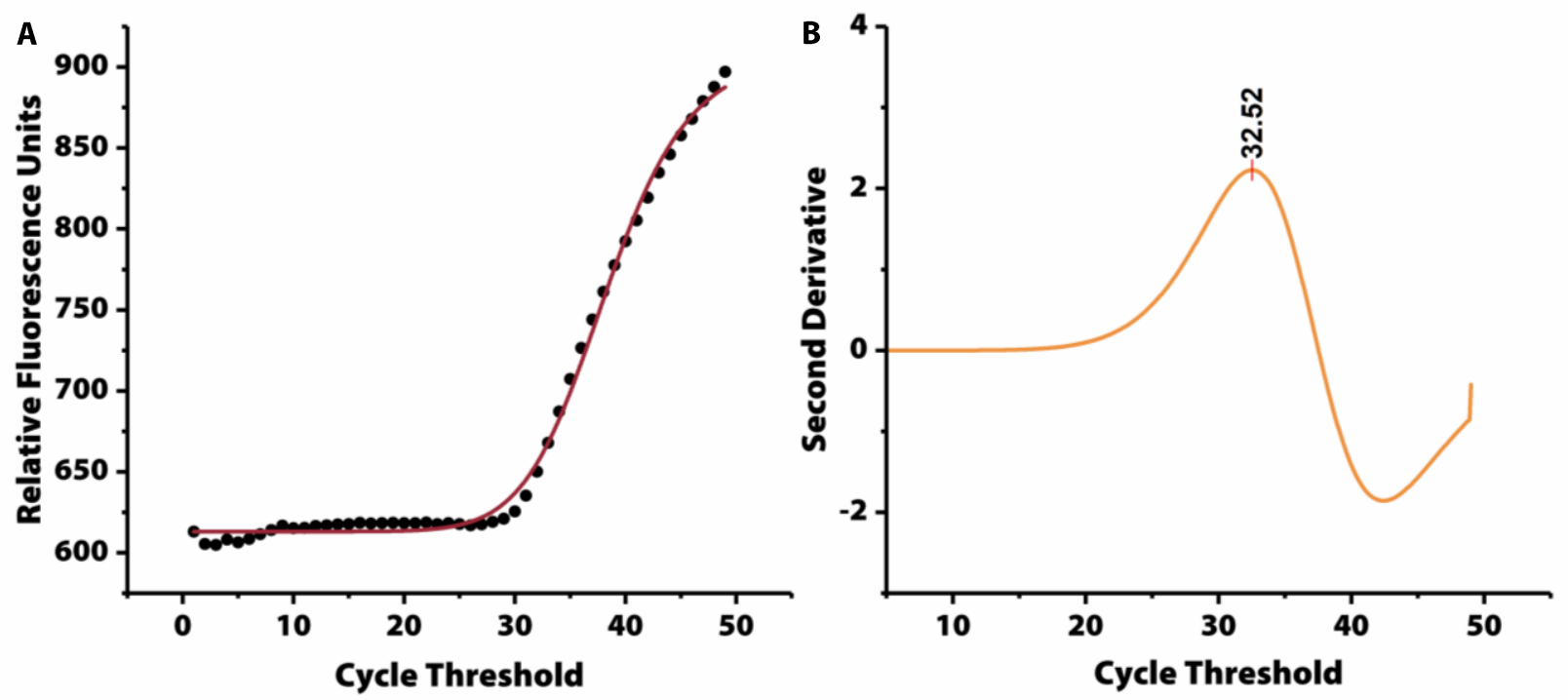

Figure s6. Ct determination using logistic fitting. (A) A plot of amplification signal (black dots) generated on the magnetofluidic device and its logistic fitting (red curve). (B) The second derivative of the logistic fitting. The x value of the highest peak depicts the Ct value.

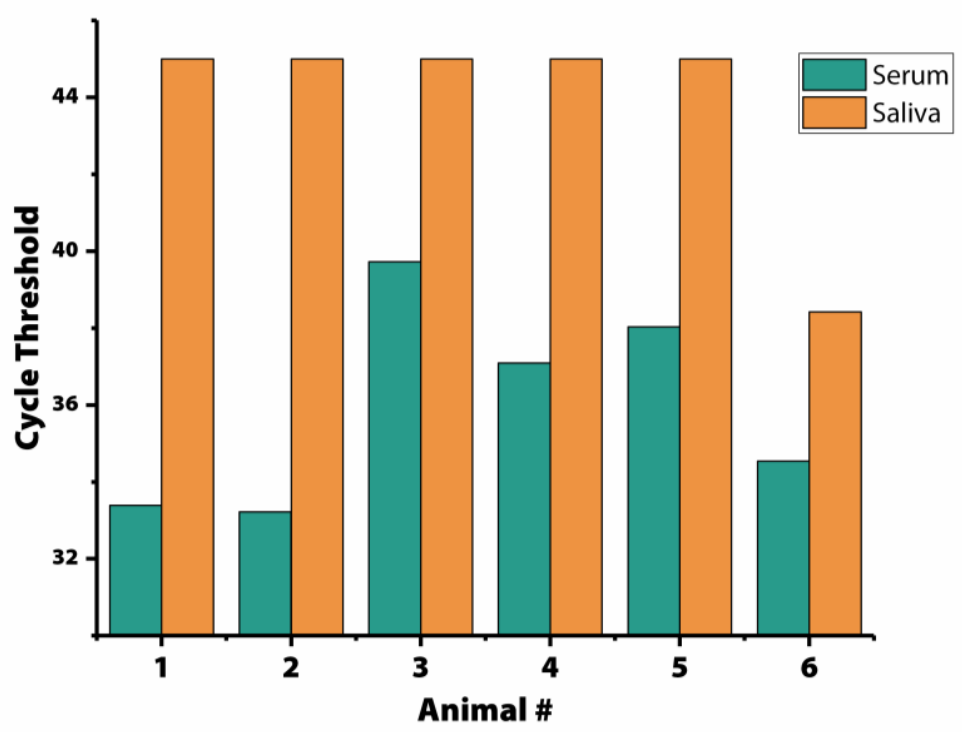

Figure s7. Sensitivity comparison of saliva and serum specimens from the same animals. Samples with no amplification are given a cycle threshold of 45. 